\title{
Negative anti-SARS-CoV-2 S antibody response following Pfizer SARS-CoV-2 vaccination in a patient on ocrelizumab
}

\author{
Mahsa Khayat-Khoei ${ }^{1} \cdot$ Sarah Conway ${ }^{1} \cdot$ Douglas A. Rubinson $^{2} \cdot$ Petr Jarolim $^{3} \cdot$ Maria K. Houtchens $^{1} \mathbb{C}$
}

Received: 5 February 2021 / Revised: 9 February 2021 / Accepted: 10 February 2021 / Published online: 27 February 2021

(c) Springer-Verlag GmbH, DE part of Springer Nature 2021

\section{Dear Sirs,}

Several vaccines for novel Coronavirus (SARS-CoV-2) are now available [1]. In Multiple Sclerosis (MS) patients on anti-CD20 therapy, blunted antibody responses to SARSCoV-2 infection [2-4] and to some vaccines have been reported [5]. However, there is no data on the immune response to mRNA vaccines against SARS-CoV-2 in patients on anti-CD20 therapies. We present a patient with MS on ocrelizumab, who received the Pfizer mRNA COVID-19 vaccine, and did not seroconvert 27 days after the second vaccine dose as measured by an FDA approved Anti-SARSCoV-2 S assay.

\section{Case presentation}

A 44-year-old Caucasian male presented 4 years ago with symptomatic myelitis and multifocal demyelinating $\mathrm{T} 2$ and $\mathrm{T} 1 \mathrm{Gadolinium}$ contrast enhancing $(\mathrm{T} 1 \mathrm{Gd}+)$ lesions throughout the brain and spinal cord. He was diagnosed with Relapsing Remitting MS (RRMS), initiated on semi-annual ocrelizumab therapy and had no evidence of disease activity (NEDA) for 3.5 years. He had been repeatedly tested for the presence of SARS-CoV-2 by RT-PCR through the institutional research program (Fig. 1). He received his first dose of SARS-CoV-2 (Pfizer) vaccine 5 months after ocrelizumab infusion, followed by the second dose 21 days later. There was a moderate post-vaccination systemic reaction

Maria K. Houtchens

mhoutchens@bwh.harvard.edu

1 Department of Neurology, Brigham and Women's Hospital, Harvard Medical School, Boston, MA, USA

2 Department of Medical Oncology, Dana-Farber Cancer Institute and Harvard Medical School, Boston, MA, USA

3 Department of Pathology, Brigham and Women's Hospital, and Harvard Medical School, Boston, MA, USA with myalgias and fatigue. He received another ocrelizumab infusion 9 days after the second dose of the vaccine (Fig. 1). The decision to receive treatment on schedule was made considering his excellent therapeutic response to date with on-label use of ocrelizumab. On the day of infusion, he had complete depletion of $\mathrm{CD} 19+$ and $\mathrm{CD} 20+$ but Immunoglobulin $\mathrm{G}(\mathrm{IgG})$ level and Absolute Lymphocyte Count (ANC) were normal (Fig. 1). At the time of infusion he tested negative for antibodies to the SARS-CoV-2 nucleoplasmid antigen (Roche Elecsys Anti-SARS-CoV-2), an assay that interrogates for a humoral immune response to SARS-CoV-2 indicating prior infection [6]. This assay does not reflect an immune response from the mRNA vaccines, directed against virus spike but not nucleocapsid protein.

27 days after the second vaccine dose and 18 days after ocrelizumab infusion, the patient was tested using the Roche Elecsys Anti-SARS-CoV-2 S assay (Fig. 1). This assay is intended for qualitative and semiquantitative detection of antibodies to the SARS-CoV-2 spike (S) protein receptorbinding domain. It is designed to detect adaptive immune response indicating prior infection, and to uniquely detect SARS-Cov-2 anti-S antibodies post mRNA vaccination [7]. Based on the manufacturer's internal studies in healthy subjects and patients infected with SARS-CoV-2 (positive RT-PCR), the assay is highly specific with a negative predictive value (NPV) of 99.98, while its sensitivity increases from $90.6 \%$ 0-7 days after confirmed infection to $96.6 \%>=15$ days after positive RT-PCR [8].

The result of this spike antibody assay was negative in our patient indicating no measurable post-vaccination immunity (negative results $<0.40 \mathrm{U} / \mathrm{mL}$ ).

\section{Discussion}

To our knowledge, this is the first report of SARS-COV-2 vaccine response in a patient with multiple sclerosis on intravenous anti-CD20 therapy. There are cases of negative 


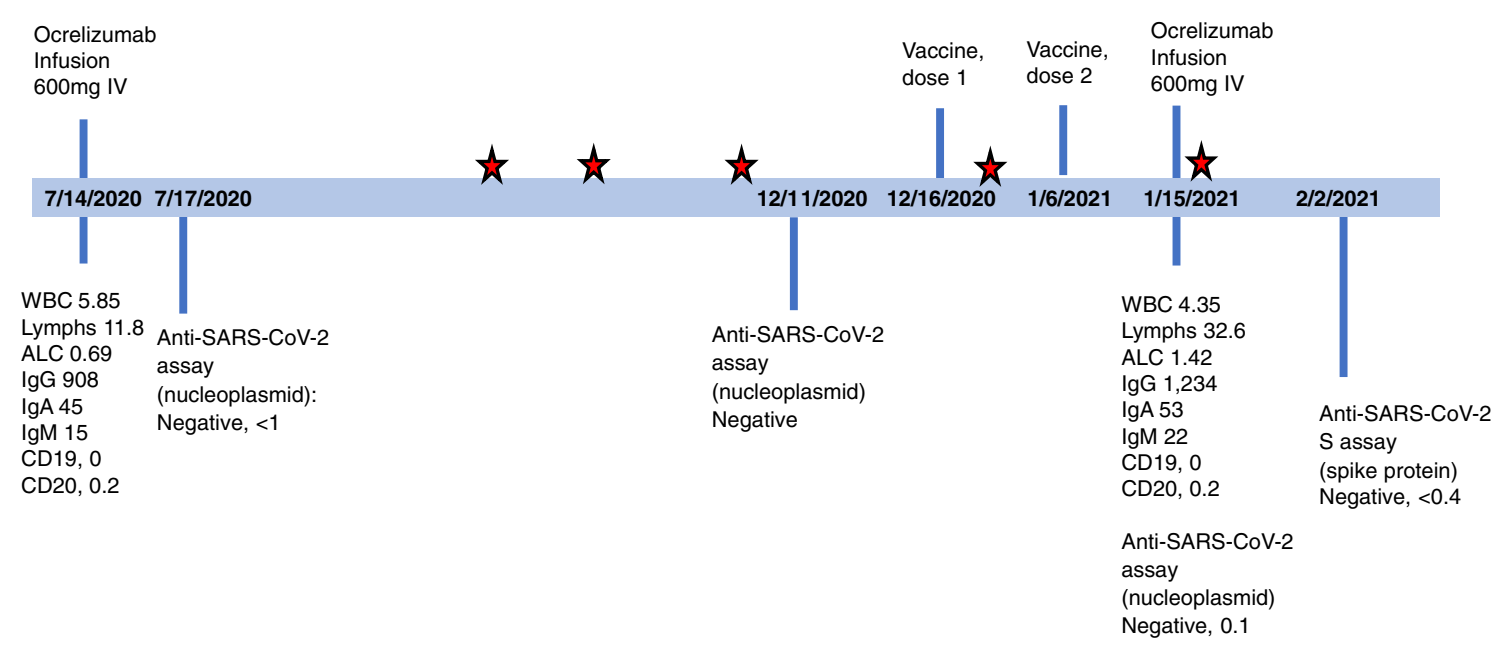

Fig. 1 Timeline of patient's ocrelizumab infusions, vaccination, SARS-CoV-2 test and laboratory results. Patient had serial SARSCoV-2 PCR tests (negative) through the institutional research program. The red stars indicate negative SARS-CoV-2 PCR tests. Reference range for Anti-SARS-CoV-2 assay (nucleoplasmid) $<1.00 \mathrm{COI}$,

SARS-CoV-2 IgG assays in MS patients with PCR-confirmed COVID-19 infection on ocrelizumab [2-4]. A recent publication of persistent anti-SARS-CoV-2 IgG antibodies following COVID19 infection in patient on ofatumumab, suggests a detectable humoral response to SARS-CoV-2 [9]. Another investigation indicates that binding titers to spike receptor-binding domain (RBD) protein as assessed on enzyme-linked immunosorbent assay (ELISA) are significantly increased on day 15 post initial 100- $\mu \mathrm{g}$ dose of mRNA-1273 vaccination [10]. Further, unpublished internal validation studies in 24 healthy volunteers for Anti-SARS-CoV-2 S assay at our institution (Dr. Petr Jarolim) suggest positive anti-spike serology after the first dose of mRNA vaccine in all subjects, with some showing titers greater than $250 \mathrm{U} / \mathrm{mL}$ (analytical measurement range).

The VELOCE trial in RRMS patients showed attenuated but present humoral responses following pneumococcal, influenza and tetanus toxoid vaccinations upon initiation of ocrelizumab [5]. Blunted immunity to hepatitis B vaccine has been observed in patients on rituximab [11, 12]. Ocrelizumab does not affect plasma cells directly and naïve B-cells generally recover significantly faster than memory B-cells [12].

Our patient did not seroconvert when tested 27 days after the second dose of the Pfizer mRNA vaccine. It is likely that antibody response would have been generated after the 1 st and the 2 nd vaccine doses, 5 months after the prior infusion, despite re-treatment with ocrelizumab on day 9 post-vaccination (Fig. 1). However, it is possible that early re-treatment with ocrelizumab may have further dampened post-vaccination immunity.
Anti-SARS-CoV-2 spike $(\mathrm{S})<0.8 \mathrm{U} / \mathrm{mL}$, WBC $4.00-10.00 \mathrm{~K} / \mathrm{uL}$, Lymphocytes $18.0-41.0 \%$, ALC $0.72-4.10 \mathrm{~K} / \mathrm{uL}$, IgG 700-1,600 mg/ dL, IgA 70-400 mg/dL, IgM 40-230 mg/dL, CD19 7.0-27.0\% lymphocytes, CD20 3.0-20.0\% lymphocytes

Our case documents undetectable humoral response after mRNA SARS-CoV-2 vaccination in a B-cell depleted patient and this is potentially concerning. However, the clinical relevance of this result is unclear as it may not indicate a complete absence of an immunity to the wild type SARS-CoV-2 infection [2, 10] In this setting, the formation of antigen-specific cytotoxic anti-viral $\mathrm{T}$ cells may help provide some protection. It is not known if the absence of Anti-SARS-CoV-2 S antibodies reflects suboptimal vaccine response and whether delaying anti-CD20 treatments to allow B cell reconstitution before administering COVID-19 mRNA vaccine may be warranted in some patients $[2,9]$

Larger studies investigating COVID-19 mRNA vaccine response in patients on anti-CD20 treatments are essential to define the optimal "vaccination window."

Author contributions statements All authors contributed to the study conception and design. The first draft of the manuscript was written by Mahsa Khayat-Khoei and all authors edited subsequent versions of the manuscript. All authors read and approved the final draft.

\section{Compliance with ethical standards}

Conflicts of interest Authors have no conflict of interest.

Ethics approval All studies performed at the Brigham and Women's Hospital are approved by the appropriate ethics committee and performed in accordance with the ethical standards laid down in the 1964 Declaration of Helsinki and its later amendments.

Consent Patient signed informed consent regarding publishing his data. 


\section{References}

1. Tregoning JS, Brown ES, Cheeseman HM et al (2020) Vaccines for COVID-19. Clin Exp Immunol 202:162-192. https://doi. org/10.1111/cei.13517

2. Thornton JR, Harel A (2020) Negative SARS-CoV-2 antibody testing following COVID-19 infection in Two MS patients treated with ocrelizumab. Mult Scler Relat Disord 44:102341. https://doi. org/10.1016/j.msard.2020.102341

3. Conte WL (2020) Attenuation of antibody response to SARS$\mathrm{CoV}-2$ in a patient on ocrelizumab with hypogammaglobulinemia. Mult Scler Relat Disord 44:102315. https://doi.org/10.1016/j. msard.2020.102315

4. Lucchini M, Bianco A, Del Giacomo P et al (2020) Is serological response to SARS-CoV-2 preserved in MS patients on ocrelizumab treatment? A case report. Mult Scler Relat Disord 44:102323. https://doi.org/10.1016/j.msard.2020.102323

5. Bar-Or A, Calkwood JC, Chognot C et al (2020) Effect of ocrelizumab on vaccine responses in patients with multiple sclerosis: The VELOCE study. Neurology 95:e1999-e2008. https://doi. org/10.1212/WNL.0000000000010380

6. Prince HE, Givens TS, Lapé-Nixon M et al (2020) Detection of SARS-CoV-2 IgG targeting nucleocapsid or spike protein by four high-throughput immunoassays authorized for emergency use. J Clin Microbiol. https://doi.org/10.1128/JCM.01742-20

7. Lerner AM, Eisinger RW, Lowy DR et al (2020) The COVID19 serology studies workshop: recommendations and challenges. Immunity 53:1-5. https://doi.org/10.1016/j.immuni.2020.06.012

8. Elecsys ${ }^{\circledR}$ Anti-SARS-CoV-2 S. In: Diagnostics. https://diagn ostics.roche.com/us/en/products/params/elecsys-anti-sars-cov2-s.html. Accessed 9 Feb 2021

9. Flores-Gonzalez RE, Hernandez J, Tornes L et al (2021) Development of SARS-CoV-2 IgM and IgG antibodies in a relapsing multiple sclerosis patient on ofatumumab. Mult Scler Relat Disord 49:102777. https://doi.org/10.1016/j.msard.2021.102777

10. Widge AT, Rouphael NG, Jackson LA et al (2021) Durability of Responses after SARS-CoV-2 mRNA-1273 Vaccination. N Engl J Med 384:80-82. https://doi.org/10.1056/NEJMc2032195

11. Richi P, Alonso O, Martín MD et al (2020) Evaluation of the immune response to hepatitis $\mathrm{B}$ vaccine in patients on biological therapy: results of the RIER cohort study. Clin Rheumatol 39:2751-2756. https://doi.org/10.1007/s10067-020-05042-2

12. Baker D, Roberts C, a. K, Pryce G, et al (2020) COVID-19 vaccine-readiness for anti-CD20-depleting therapy in autoimmune diseases. Clin Exp Immunol 202:149-161. https://doi. org/10.1111/cei.13495 